STATE OF ALASKA

DEPARTMENT OF NATURAL RESOURCES

DIVISION OF GEOLOGICAL AND GEOPHYSICAL SURVEYS

Bill Sheflield, Governor

Esther C. Wunnicke, Commissioner

Ross G. Schaff, Director and State Geologist

May 1986

This report is a preliminary publication of DGGS.

The author is solely responsible lor its content and will appreciate candid comments on the accuracy of the data as well as suggestions to improve the report.

\author{
Report of Invest1gations 86-1 \\ PRELIMINARY GRAVITY DATA OF THE \\ MINCHUMINA BASIN, SOUTHCENTRAL ALASKA
}

By

J.F. Meyer and D.L. Krouskop 
STATE OF ALASKA

Department of Natural Resources

DIVISION OF GEOLOGICAL \& GEOPHYSICAL SURVEYS

Accordlng to Alaska Statute 41, the Alaska Division of Geological and Geophyglcal Surveys is charged with conducting 'geologlcal and geophysical surveys to determine the potential of Alagkan land for production of metals, minerals, fuels, and geothermal resources; the locations and supplies of ground water and construction materials; the potentlal geologic hazarda to bulldings, roads, bridges, and other installatlong and structures; and shall conduct such other surveys and investigations as w111 advaace knowledge of the geology of Alaska.'

In addition, the Division of Geological and Geophyalcal Surveys shall collect, record, evaluate, and distribute data on the quantity, quality, and location of underground, surface, and coastal water of the state; publish or have published data on the water of the state and require that the results and findings of surveys of water quality, quantity, and location be filed; require that water-well contractors flle basic water and aquifer data, including but not limited to well location, estimated elevation, welldriller's logs, pumping tests, flow measurements, and water-quality determinations; accept and spend funds for the purposes of this section, AS 41.08.017 and 41.08.035, and enter 1nto agreements with 1ndividuals, pub11c or private agencies, commities, private industry, and state and federal agencles; collect, record, evaluate, archive, and distribute data on seismic events and englneering geology of the state; and 1deatify and 1aform public officlals and 1ndustry about potential selamlc hazards that wight affect development in the state.

Administrative functions are performed under the direction of the State Geologiat, who maintains his office in Anchorage. DGGS offlcea are locaced at:

.794 Untvergity Ave.
(Basement)
Ea1rbanks, 99709
(907) $474-7147$
.400 W11loughby Center
(3rd f1.)
Juneau, 99801
(907) $465-3400$

This report is for sale by DGGS for spected at the following locations. Ma11 Gairbanks office.

\author{
.3601 C St. (8th Fl.) \\ P.O Box 7028 \\ Anchorage, 99510 \\ (907) 561-2020 \\ . F1sh Hetchery Road \\ R.0. Box 772116 \\ Eagle River, 99577 \\ (907) 688-3555
}

$\begin{array}{ll}.794 \text { University Ave. } & .3601 \mathrm{CSt} .(10 \mathrm{th} \mathrm{fl} .) \\ \text { (Basement) } & \text { P.0. Box } 7005 \\ \text { Fa1rbanks, } 99709 & \text { Anchorage, } 99510 \\ & \\ 400 \text { W11loughby Center } & \text {. P.0. Box } 7438 \\ \text { (4th fl.) } & \text { State Off1ce B1dg. } \\ \text { Juneau, } 99801 & \text { Ketchikan, } 99901\end{array}$




\section{CONTENTS}

$\underline{\text { Page }}$

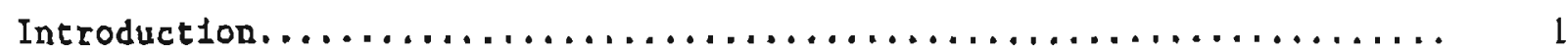

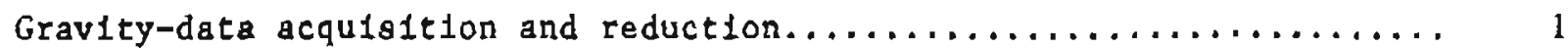

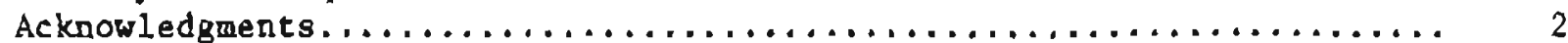

References c1ted...................................... 2

\section{TABLE}

Table L. Data for new gravity stations occupled in the Minchumina Basin, Alaska.............................

\section{SHEETS}

Sheet 1. Free-air gravity map of the Minchumina Basin, Alaska...... Pocket 2. Complete Bouguer gravity map of the Minchumina Basia, Alaska.................................. Pocket 


\title{
PRELIMINARY GRAVITY DATA OF THE MINCHUMINA BASIN, SOUTHCENTRAL ALASKA
}

\author{
J.F. Meyer and D.L. Krouskop 1
}

\section{INTRODUCTION}

The Minchumina Basin is a subarctic playn located southwest of Lake Minchumins in the upper portion of the Kuskokwim River drainage area. Although this basin is in the intital stages of exploration, it is one of the few areas in interior Alaska where oil and gas wight be discovered. The basin is bound on the southeast by the Denall fault system, Including the Farewe11 fault, and on the northwest by the N1xon Fork fault system.

From 1981 to 1983, the Division of Geological and Geophysical surveys (DGGS) conducted a surface gravity survey that complements and extends gravity data obtained from the U.S. Geolog1cal Survey (USGS). Thts report presents the reduced data in tabular form and the contoured free-air gravity map and the terraln-corrected Bouguer grav1ty map (sheets 1 and 2 ).

\section{GRAVITY-DATA ACQUISITION AND REDUCTION}

The gravity analysis of this area is based on information we collected from 510 statlons during the summers of 1981 and 1983 and information from 387 stations previously obtalned by D.F. Barnes (USGS). The latter data were provided as digitized gravity fleld data on magnetic tape from the USGS gravity library. Some USGS stations were reoccupied to compare data sets collected by both agencles.

Datum control for all gravity values was provided by the USGS Alaskan Gravity Base Station Network (Barnes, 1968; 1972) and was adjusted to the new absolute datum of the International Gravity Standardization Net 1971 (Morel11 and others, 1974). The new data were tied lnto this network by establishing two fleld base stations: one at Farewell landing Fleld and the other at Farewell Lake Lodge.

A Lacoste and Romberg gravity meter (G-507) was used to establish the new gravlty stations. During the fleld surveys, the gravity meter appeared to function properly, and a maximum drift of $0.5 \mathrm{mgal} / \mathrm{day}$ indicates there were no apparent tares in the data.

Three altimeters (American Paulin Model $T-5$ ) were used to determine elevations for the new surveys. The three readings were averaged at each station and corrected for diumal barometrlc varlations using data from a base-station micro-barograph (American Paulin Model SMB-5) located at Farewe 11 Lake Lodge. Where feasible, the gravity stations were located at U.S. Coast and Geodet1c Survey Vertical Angle Benchmarks (VABM) for elevation control. Temperature and drift corrections were also applied and ylelded elevations with an accuracy of $\pm 30 \mathrm{ft}$. Many types of elevation control were

IDGGS, P.0. Box 7028, Anchorage, Alaska 99510. 
used to reduce the USGS data. Some elevations were calculated from field altimetry measurements; others were calculated from river gradients derived from topographic maps. The altimetry analysis 1ndicates that 90 percent of the data are accurate to $\pm 50 \mathrm{ft}$ (Barnes, 1977).

Gravity reductions were run on all data (1ncluding the data obtalned from the. USGS) using standard technlques, lncluding a latitude correction defined by the new ellipgold obtalned from the 1967 Geodet1c Reference System (International Assoctation of Geodesy, 1971). A density of $2.62 \mathrm{gm} / \mathrm{cc}$, which was used for the Bouguer correction over the entire area, was obtalned by averaging the densities of 195 representative rocks from the area (Btandard deviation $\pm 0.21 \mathrm{gm} / \mathrm{cc}$ ). Terrain corrections were calculated using a USGS computer program and digltized topographic data. Terra1n corrections varled Erom 0 to 0.5 mgal 1 a the basin to 24.71 mgal in the mountaing. To check the accuracy of the computer corrections, hand-calculated terra1n corrections were determined for several stations and compared to the computer corrections. The comparison was excellent, with a maximum difference of $0.25 \mathrm{mgal}$. The precision of the Bouguer anomaly values is primarlly dependent on the elevation and $1 \mathrm{~s}$ estimated at $\pm 3 \mathrm{mgal}$ based on the $50-\mathrm{ft}$ accuracy of the older data. Data for the new gravity gtations are given in table 1; elevations represent the altimeter elevation. For comparison with other data sets, the complete Bouguer anomaly $1 \mathrm{~s}$ glven for a density of $2.67 \mathrm{gm} / \mathrm{cc}$ as we11 as a calculated density of $2.62 \mathrm{gm} / \mathrm{cc}$.

A free-alr gravtty map and a terraln-corrected Bouguer gravity map were produced 1ncorporating both data sets (sheets 1 and 2 ). Because the new and old gravity values closely agree for those stations occupled by the USGS and DGGS, no problems arose tn contouring the data.

\section{ACKNOWLEDGMENTS}

We gratefully acknowledge B.K. W1lson (DGGS) for h1s assistance in collecting and reducing this data and the cooperation of D.F. Barnes in maklng his date avallable to us. We also thank S.M. Weum (DGGS) for revlewing the manuscript.

\section{REFERENCES CITED}

Barnes, D.E., 1968, Alaska grav1ty bese statlon network: U.S. Geologlca1 Survey Open-f1le report, $21 \mathrm{p}$.

1972, Southeast Alaska gravity base station network: U.S. Geological Survey Open-f1le report, $40 \mathrm{p}$. 1977, Bouguer gravity map of Alaska; U.S. Geological Survey Geophyical Invest1gations Map GP-913, scale 1:2,500,000.

International Association of Geodesy, 1971, Geodet1c reference system, 1967: Parta, Bureau Central de l'Association Internationale de Geodesie, Special Publication 3, 116 p.

Morel11, Carlo, Gantar, C., Honkasala, Tauno, McConnell, R.K., Tanner, J.G., Szabo, Bela, Uot1la, U.A., and Whalen, C.T., 1974, The International Gravity Standardization Net 1971 (IGSN 71): Par1s, Bureau Central de l'Association Internationale de Geodesie, Special Publication 4, $194 \mathrm{p}$. 
Table 1. Data for new gravity stations occupled in the Minchumina Basin, Alaska.

\begin{tabular}{|c|c|c|c|c|c|c|c|c|}
\hline \multicolumn{2}{|c|}{ Station } & Latitude & \multicolumn{2}{|r|}{$\begin{array}{l}\text { Elev } \\
(\mathrm{ft})\end{array}$} & Observed & FAA & $\mathrm{CBA}(2.62)$ & $\mathrm{CBA}(2.67)$ \\
\hline$M$ & I & $62 \quad 28.74$ & $153 \quad 30.22$ & 1110 & 981998.37 & -3.82 & -38.67 & -39.33 \\
\hline M & 2 & $62 \quad 29.20$ & 15330.66 & 1100 & 982001.37 & -2.29 & -36.95 & -37.61 \\
\hline M & 3 & $62 \quad 29.57$ & 15331.00 & 1081 & 982004.87 & -1.09 & -35.31 & -35.96 \\
\hline M & 4 & 6230.14 & 15331.78 & 1062 & 982007.87 & -0.49 & -34.49 & -35.14 \\
\hline M & 5 & $62 \quad 30.92$ & $153 \quad 31.55$ & 1045 & 982008.69 & -2.32 & -36.11 & -36.76 \\
\hline M & 6 & 6231.52 & $153 \quad 32.92$ & 1042 & 982010.62 & -1.38 & -35.34 & -35.98 \\
\hline M & 7 & 6232.14 & $153 \quad 33.98$ & 1025 & 982014.19 & -0.18 & -33.71 & -34.35 \\
\hline M & 8 & $62 \quad 32.72$ & $153 \quad 34.95$ & 1020 & 982019.12 & 3.57 & -29.88 & -30.52 \\
\hline M & 9 & $62 \quad 33.82$ & 15335.02 & 1004 & 982024.37 & 6.01 & -27.11 & -27.74 \\
\hline M & 10 & 6234.88 & 15335.74 & 988 & 982031.37 & 10.10 & -22.54 & -23.16 \\
\hline$M$ & 11 & 6235.65 & 15337.10 & 965 & 982037.62 & 13.17 & -18.70 & -19.31 \\
\hline M & 12 & 6236.41 & 15337.48 & 949 & 982041.87 & 15.08 & -16.35 & -16.95 \\
\hline$M T$ & 23 & $62 \quad 36.74$ & $153 \quad 39.89$ & 924 & 982042.81 & 13.15 & -17.45 & -18.04 \\
\hline M 1 & 14 & 6237.09 & 15341.50 & 913 & 982047.12 & 16.10 & -14.14 & -14.72 \\
\hline $\mathrm{M}$ & 15 & $\begin{array}{ll}62 & 37.57\end{array}$ & 15343.41 & 892 & 982048.63 & 14.97 & -14.50 & -15.06 \\
\hline$M$ & 16 & 6238.47 & 15343.95 & 879 & 982050.87 & 35.01 & -14.20 & -14.76 \\
\hline M 1 & 17 & 6239.41 & 15345.09 & 867 & 982053.37 & 15.18 & -13.65 & -14.20 \\
\hline M 1 & 18 & 6240.11 & 15346.09 & 849 & 982054.81 & 14.05 & -14.19 & -14.73 \\
\hline M 1 & 19 & 6241.02 & 15347.42 & 821 & 982058.81 & 14.26 & -12.95 & -13.47 \\
\hline$M 2$ & 20 & 6241.85 & 15347.77 & 819 & 982060.62 & 24.84 & -12.42 & -12.94 \\
\hline $\mathrm{M} 2$ & 21 & 6242.51 & 15348.20 & 803 & 982064.81 & 16.69 & -10.06 & -10.57 \\
\hline $\mathrm{M} 2$ & 22 & $62 \quad 43.33$ & 15349.42 & 785 & 982067.50 & 16.69 & -9.46 & -9.96 \\
\hline $\mathrm{M} 2$ & 23 & $62 \quad 43.86$ & 15350.25 & 772 & 982073.19 & 20.54 & -5.19 & -5.68 \\
\hline $\mathrm{M} 2$ & 24 & 6244.45 & 15350.77 & 754 & 982079.13 & 24.06 & -1.03 & -1.51 \\
\hline $\mathrm{M} 2$ & 25 & 6244.94 & 15352.98 & 731 & 982064.87 & 7.09 & -17.27 & -17.73 \\
\hline $\mathrm{M}$ & 26 & $62 \quad 45.67$ & 15354.75 & 718 & 982064.69 & 4.70 & -19.27 & -19.73 \\
\hline M2 2 & 27 & $52 \quad 46.51$ & $153 \quad 56.52$ & 702 & 982065.12 & 2.63 & -20.80 & -21.25 \\
\hline$M 2$ & 28 & $62 \quad 47.23$ & 15357.14 & 677 & 982066.87 & 1.18 & -21.41 & -21.84 \\
\hline M 2 & 29 & 6247.68 & 15358.37 & 669 & 982067.87 & 0.85 & -21.46 & -21.89 \\
\hline$M 3$ & 30 & 6248.41 & 15359.18 & 641 & 982069.81 & -0.83 & -22.20 & -22.61 \\
\hline$M 3$ & 31 & 6248.82 & 15359.21 & 624 & 982071.87 & -0.81 & -21.61 & -22.01 \\
\hline$M 3$ & 32 & 6249.44 & 15359.86 & 618 & 982074.38 & 0.44 & -20.20 & -20.59 \\
\hline$M 3$ & 33 & 6250.24 & 1540.53 & 597 & 982076.37 & -0.51 & -20.45 & -20.83 \\
\hline$M 3$ & 34 & 6251.15 & 1540.18 & 587 & 982078.87 & -0.15 & -19.75 & -20.12 \\
\hline$M 3$ & 35 & 6252.01 & 1540.27 & 568 & 982082.12 & 0.21 & -18.75 & -19.11 \\
\hline$M=$ & 36 & 6252.34 & $154 \quad 1.69$ & 562 & 982084.50 & 1.68 & -17.08 & -17.44 \\
\hline$M 3$ & 37 & 6253.19 & 1542.11 & 549 & 982086.31 & 1.21 & -17.11 & -17.46 \\
\hline$M 3$ & 38 & 6253.90 & $154 \quad 3.92$ & 544 & 982087.31 & 0.85 & -17.32 & -17.66 \\
\hline$M 3$ & 39 & 6254.62 & $154 \quad 5.33$ & 525 & 982088.62 & -0.54 & -18.08 & -18.41 \\
\hline$M$ & 40 & 6255.41 & 1546.43 & 520 & 982092.31 & 2.77 & -15.60 & -15.93 \\
\hline M & 41 & 6256.29 & $154 \quad 7.22$ & 512 & 982093.69 & 1.32 & -15.79 & -16.11 \\
\hline$M$ & 42 & 6257.01 & 1548.54 & 498 & 982092.87 & -1.67 & -18.31 & -18.63 \\
\hline$M$ & 43 & 6257.70 & $154 \quad 10.00$ & 493 & 982092.12 & -3.85 & -20.33 & -20.65 \\
\hline$M$ & 44 & 6258.23 & 15411.62 & 491 & 982091.19 & -5.56 & -21.98 & -22.29 \\
\hline$M$ & 45 & 6258.91 & $154 \quad 13.09$ & 481 & 982091.19 & -7.35 & -23.43 & -23.74 \\
\hline M & 46 & $62 \quad 59.48$ & $154 \quad 14.56$ & 474 & 982093.62 & -6.33 & -22.17 & -22.48 \\
\hline
\end{tabular}

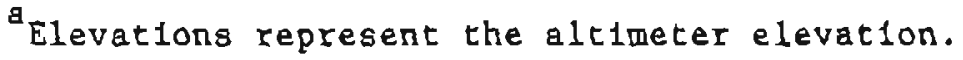


Table 1 (con.)

\begin{tabular}{|c|c|c|c|c|c|c|c|c|c|}
\hline \multirow{2}{*}{$\begin{array}{l}\text { Station } \\
\text { M } 47\end{array}$} & \multicolumn{2}{|c|}{ Latitude } & \multicolumn{2}{|c|}{ Long1tude } & $\begin{array}{l}\text { Elev } \dot{~} \\
(f t)^{8}\end{array}$ & Observed & FAA & $\operatorname{CBA}(2.62)$ & $\operatorname{CBA}(2.67)$ \\
\hline & 63 & 0.21 & 154 & 16.54 & 459 & 982095.81 & -6.41 & -21.75 & -22.04 \\
\hline M 48 & 63 & 0.62 & 154 & 18.14 & 465 & 982100.31 & -1.89 & $=17.42$ & $-17,72$ \\
\hline M 49 & 62 & 59.89 & 154 & 19.48 & 462 & 982099.19 & -2.32 & -17.75 & -18.05 \\
\hline M 50 & 62 & 20.64 & 154 & 3.48 & 1722 & 981962.00 & 27.43 & -24.27 & -25.26 \\
\hline M 51 & 62 & 22.09 & 154 & 4.99 & 1640 & 981966.87 & 22.80 & -24.21 & -25.10 \\
\hline M 52 & 62 & 21.27 & 154 & 4.37 & 1661 & 981965.00 & 23.89 & -24.17 & -25.08 \\
\hline M 53 & 62 & 22.92 & 154 & 5.56 & 1583 & 981973.87 & 23.45 & -23.72 & -24.62 \\
\hline M 54 & 62 & 23.60 & 154 & 6.82 & 1529 & 981981.31 & 24.94 & -21.75 & -22.64 \\
\hline$M 55$ & 62 & 24.34 & 154 & 7.70 & 1491 & 981982.88 & 22.06 & -24.92 & -25.82 \\
\hline$M 56$ & 62 & 25.05 & 154 & 8.77 & 1454 & 981984.31 & 19.05 & -27.75 & -28.65 \\
\hline M 57 & 62 & 25.51 & 154 & 10.37 & 1399 & 981983.31 & 12.31 & -33.09 & -33.95 \\
\hline M 58 & 62 & 26.22 & 154 & 11.26 & 1364 & 981984.37 & 9.20 & -35.41 & -36.27 \\
\hline M 59 & 62 & 27.06 & 154 & 11.86 & 1317 & 981988.12 & 7.49 & -35.80 & -36.62 \\
\hline$M 60$ & 62 & 27.96 & 154 & 12.68 & 1276 & 981993.12 & 7.54 & -34.51 & -35.32 \\
\hline$M 61$ & 62 & 28.85 & 154 & 13.49 & 1242 & 981998.37 & 8.51 & -32.63 & -33.42 \\
\hline M 62 & 62 & 29.37 & 154 & 14.68 & 1192 & 982005.31 & 10.03 & -29.46 & -30.21 \\
\hline$M 63$ & 62 & 29.95 & 154 & 16.17 & 1142 & 982018.62 & 17.88 & -20.01 & -20.73 \\
\hline M 64 & 62 & 30.69 & 154 & 17.07 & 1105 & 982025.13 & 20.05 & -16.73 & -17.43 \\
\hline M 65 & 62 & 31.43 & 154 & 18.35 & 1066 & 982032.50 & 22.88 & -12.66 & -13.33 \\
\hline M 66 & 62 & 32.20 & 154 & 19.27 & 1025 & 982036.81 & 22.36 & -11.83 & -12.48 \\
\hline$M 67$ & 62 & 32.85 & 154 & 20.15 & 982 & 982041.87 & 22.63 & -10.14 & -10.76 \\
\hline M 68 & 62 & 33.49 & 154 & 21.74 & 933 & 982043.81 & 19.13 & -12.00 & -12.60 \\
\hline M 69 & 62 & 34.24 & 154 & 22.26 & 880 & 982048.50 & 17.85 & -11.52 & -12.08 \\
\hline$M 70$ & 62 & 34.94 & 154 & 22.28 & 854 & 982052.37 & 18.44 & -10.06 & -10.60 \\
\hline$M 71$ & 62 & 35.27 & 154 & 24.01 & 812 & 982054.87 & 16.67 & -10.44 & -10.96 \\
\hline M 72 & 62 & 35.81 & 154 & 25.61 & 765 & 982058.87 & 15.52 & -10.02 & -10.51 \\
\hline$M 73$ & 62 & 36.66 & 154 & 26.33 & 722 & 982063.69 & 15.23 & -8.88 & -9.34 \\
\hline$M 74$ & 62 & 37.43 & 154 & 27.59 & 683 & 982068.87 & 15.76 & -7.04 & -7.48 \\
\hline M 75 & 62 & 37.79 & 154 & 29.37 & 647 & 982071.31 & 14.30 & -7.31 & -7.72 \\
\hline M 76 & 62 & 38.43 & 154 & 30.45 & 621 & 982074.31 & 14.05 & -6.69 & -7.08 \\
\hline M 77 & 62 & 39.16 & 154 & 31.85 & 586 & 982075.50 & 11.13 & -8.44 & -8.81 \\
\hline M 78 & 62 & 39.88 & 154 & 32.60 & 561 & 982075.69 & 8.10 & -10.63 & -10.99 \\
\hline$M 79$ & 62 & 40,40 & 154 & 34.01 & 544 & 982076.50 & 6.62 & -11.54 & -11.89 \\
\hline M 80 & 62 & 41.25 & 154 & 35.08 & 523 & 982076.50 & 3.64 & -13.82 & -14.15 \\
\hline M 81 & 62 & 42.01 & 154 & 35.94 & 502 & 982075.37 & -0.36 & -17.13 & -17.45 \\
\hline M 82 & 62 & 42.82 & 154 & 36.82 & 495 & 982072.87 & -4.50 & -21.04 & -21.35 \\
\hline$M 83$ & 62 & 43.73 & 154 & 37.44 & 478 & 982071.12 & -9.07 & -25.04 & -25.35 \\
\hline M 84 & 62 & 44.39 & 154 & 38.26 & 473 & 982067.81 & -13.71 & -29.51 & -29.82 \\
\hline M 85 & 62 & 27.85 & 153 & 30.25 & 1167 & 981994.50 & -1.20 & -37.65 & -38.34 \\
\hline M 86 & 62 & 27.18 & 153 & 29.78 & 1188 & 981991.37 & -1.50 & -38.05 & -38.75 \\
\hline M 87 & 62 & 26.36 & 153 & 29.41 & 1202 & 981987.31 & -3.30 & -39.90 & -40.60 \\
\hline$M 88$ & 62 & 25.43 & 153 & 29.02 & 1226 & 981980.87 & -6.25 & -42.42 & -43.11 \\
\hline$M 89$ & 62 & 24.75 & 153 & 28.79 & 1246 & 981978.12 & -6.34 & -42.45 & -43.14 \\
\hline$M 90$ & 62 & 24.03 & 153 & 27.92 & 1267 & 981970.81 & -10.83 & -45.31 & -45.97 \\
\hline$M 91$ & 62 & 23.12 & 153 & 27.89 & 1277 & 981966.69 & -12.84 & -48.31 & -48.98 \\
\hline$M 92$ & 62 & 22.24 & 153 & 28.12 & 1289 & 981965.81 & -11.55 & -48.47 & -49.17 \\
\hline$M 93$ & 62 & 21.55 & 153 & 27.54 & 1309 & 981962.00 & -12.56 & -49.56 & -50.27 \\
\hline
\end{tabular}


Table 1 (con.)

\begin{tabular}{|c|c|c|c|c|c|c|c|c|c|}
\hline $10 \pi$ & Lat & titude & Lon & g1tude & $\begin{array}{l}\text { Elev } \dot{~} \\
(\mathrm{ft})^{\mathrm{a}}\end{array}$ & Observed & FAA & $\mathrm{CBA}(2.62)$ & $\mathrm{CBA}(2.67$ \\
\hline M 94 & 62 & 20.84 & 153 & 26.16 & 1325 & 981957.19 & -15.00 & -51.85 & -52.55 \\
\hline$M 95$ & 62 & 20.13 & 153 & 25.30 & 1343 & 981956.12 & -13.53 & -50.41 & -51.11 \\
\hline$M 96$ & 62 & 19.52 & 153 & 24.00 & 1360 & 981950.19 & -17.02 & -53.37 & -54.06 \\
\hline M 97 & 62 & 18.70 & 153 & 23.05 & 1377 & 981948.19 & -16.42 & -53.89 & -54.60 \\
\hline$M 98$ & 62 & 17.84 & 153 & 22.85 & 1395 & 981947.62 & -14.28 & -52.80 & -53.53 \\
\hline M 99 & 62 & 16.94 & 153 & 22.44 & 1412 & 981947.81 & -11.32 & -51.28 & -52.04 \\
\hline M100 & 62 & 16.06 & 153 & 22.34 & 1430 & 981940.69 & -15.64 & -55.52 & -56.28 \\
\hline M101 & 62 & 46.99 & 154 & 46.16 & 433 & 982075.31 & -13.14 & -27.60 & -27.88 \\
\hline$M 102$ & 62 & 47.56 & 154 & 47.78 & 432 & 982075.50 & -13.69 & -28.14 & -28.41 \\
\hline M103 & 62 & 48.31 & 154 & 48.55 & 424 & 982077.37 & -13.52 & -27.69 & -27.96 \\
\hline M104 & 62 & 49.13 & 154 & 49.75 & 423 & 982079.13 & -12.91 & -27.05 & -27.32 \\
\hline M105 & 62 & 50.19 & 154 & 48.73 & 415 & 982080.88 & -13.10 & -26.97 & -27.23 \\
\hline M106 & 62 & 50.97 & 154 & 49.61 & 410 & 982082.50 & -12.98 & -26.58 & $-26,94$ \\
\hline M107 & 62 & 51.74 & 154 & 50.21 & 407 & 982085.37 & -11.28 & -24.88 & -25.14 \\
\hline M108 & 62 & 52.62 & 154 & 50.92 & 396 & 982088.19 & -10.64 & -23.88 & -24.13 \\
\hline M109 & 62 & 53.32 & 154 & 51.92 & 396 & 982091.62 & -8.09 & -21.33 & -21.59 \\
\hline M110 & 62 & 54.16 & 154 & 52.86 & 390 & 982092.12 & -9.21 & -22.25 & -22.50 \\
\hline M111 & 62 & 55.02 & 154 & 53.83 & 388 & 982093.81 & -8.71 & -21.68 & -21.93 \\
\hline$M 112$ & 62 & 55.78 & 154 & 54.11 & 385 & 982095.69 & -8.01 & -20.88 & -21.12 \\
\hline $\mathrm{MI13}$ & 62 & 56.40 & 154 & 53.14 & 384 & 982097.19 & -7.38 & -20.21 & -20.46 \\
\hline M114 & 62 & 57.16 & 154 & 52.56 & 384 & 982098.19 & -7.29 & -20.11 & -20.36 \\
\hline $\mathrm{M1} 15$ & 62 & 58.07 & 154 & 52.96 & 384 & 982100.19 & -6.45 & -19.27 & -19.51 \\
\hline$M 116$ & 62 & 58.94 & 154 & 53.36 & 379 & 982102.31 & -5.86 & $-18,51$ & -18.75 \\
\hline$M 117$ & 62 & 59.74 & 154 & 54.23 & 379 & 982104.31 & -4.79 & -17.42 & -17.66 \\
\hline$M 118$ & 62 & 0.07 & 154 & 37.49 & 1350 & 981956.19 & 12.33 & -32.19 & -33.04 \\
\hline Ml19 & 62 & 0.96 & 154 & 37.75 & 1326 & 981959.62 & 12.36 & -31.31 & -32.14 \\
\hline ML20 & 62 & 1.66 & 154 & 38.87 & 1313 & 981961.62 & 12.24 & -30.90 & -31.72 \\
\hline M121 & 62 & 2.35 & 154 & 39.97 & 1288 & 981965.50 & 12.94 & -29.50 & -30.31 \\
\hline M122 & 62 & 3.00 & 154 & 40.59 & 1279 & 981970.87 & 16.66 & -25.48 & -26.28 \\
\hline MI23 & 62 & 3.74 & 154 & 41.52 & 1262 & 981974.31 & 17.58 & -24.00 & -24.79 \\
\hline$M 124$ & 62 & 4.53 & 154 & 41.13 & 1235 & 981976.69 & 16.40 & -24.26 & -25.04 \\
\hline M125 & 62 & 5.33 & 154 & 40.55 & 1224 & 981977.87 & 15.59 & -24.63 & -25.40 \\
\hline Ml26 & 62 & 5.99 & 154 & 40.10 & 1204 & 981976.69 & 11.65 & -27.90 & -28.66 \\
\hline M127 & 62 & 6.82 & 154 & 39.92 & 1188 & 981976.37 & 8.82 & -30.13 & -30.88 \\
\hline$M 128$ & 62 & 7.56 & 154 & 40.09 & 1170 & 981975.37 & 5.18 & -33.13 & -33.86 \\
\hline M129 & 62 & 8.48 & 154 & 39.75 & 1145 & 981977.37 & 3.69 & -33.66 & -34.37 \\
\hline M130 & 62 & 9.32 & 154 & 39.74 & 1133 & 981976.87 & 0.99 & -35.84 & -36.54 \\
\hline$M 131$ & 62 & 10.05 & 154 & 40.69 & 1117 & 981974.81 & -3.51 & -39.93 & -40.62 \\
\hline $\mathrm{M} 132$ & 62 & 10.78 & 154 & 41.56 & 1096 & 981974.62 & -6.56 & -42.34 & -43.02 \\
\hline M133 & 62 & 11.52 & 154 & 42.25 & 1095 & 981978.81 & -3.41 & -39.16 & -39.84 \\
\hline$M 134$ & 62 & 12.23 & 154 & 42.99 & 1058 & 981980.37 & -6.17 & -40.74 & -41.40 \\
\hline M135 & 62 & 12.92 & 154 & 43.61 & 1053 & 981982.62 & -5.30 & -39.69 & -40.35 \\
\hline M136 & 62 & 13.70 & 154 & 44.26 & 1038 & 981984.69 & -5.58 & -39.54 & -40.19 \\
\hline M137 & 62 & 14.40 & 154 & 45.29 & 1018 & 981991.31 & -1.69 & -35.18 & -35.82 \\
\hline$M 138$ & 62 & 15.38 & 154 & 45.70 & 994 & 981996.50 & 0.00 & -32.79 & -33.42 \\
\hline M139 & 62 & 16.02 & 154 & 46.99 & 989 & 981999.50 & 1.72 & -31.00 & -31.63 \\
\hline$M 140$ & 62 & 16.76 & 154 & 48.08 & 958 & 982002.37 & 0.78 & -31.00 & -31.61 \\
\hline
\end{tabular}


Table I (con.)

\begin{tabular}{|c|c|c|c|c|c|c|c|}
\hline tation & Lat1tude & Longltude & $\begin{array}{l}E l e v_{a} \\
(f t)^{B}\end{array}$ & Observed & FAA & $\operatorname{CBA}(2.62)$ & $\mathrm{CBA}(2.6$ \\
\hline$M 141$ & $62 \quad 17.40$ & 15449.27 & 940 & 982007.19 & 3.09 & -28.10 & $-28,70$ \\
\hline$M 142$ & $62 \quad 17.99$ & 15450.52 & 922 & 982010.12 & 3.55 & -27.06 & -27.65 \\
\hline$M 143$ & 6218.62 & 15451.83 & 913 & 982009.50 & 1.38 & -28.99 & -29.57 \\
\hline$M 144$ & 6219.24 & 15452.96 & 903 & 982009.12 & -0.78 & -30.80 & -31.38 \\
\hline $\mathrm{Ml45}$ & $62 \quad 20.10$ & 15453.75 & 885 & 982011.81 & -0.88 & -30.24 & -30.80 \\
\hline $\mathrm{M} 146$ & 6220.71 & 15454.71 & 868 & 982014.37 & -0.60 & -29.48 & -30.03 \\
\hline$M 147$ & 6221.65 & 15455.04 & 852 & 982018.31 & 0.63 & -27.75 & -28.29 \\
\hline M148 & 6222.46 & $154 \quad 55.28$ & 826 & 982021.37 & 0.31 & -27.20 & -27.73 \\
\hline MI49 & $62 \quad 23.24$ & 15456.18 & 817 & 982023.38 & 0.55 & -26.64 & -27.16 \\
\hline MI50 & 6224.05. & 15456.94 & 808 & 982025.87 & 1.14 & -25.77 & -26.28 \\
\hline M151 & 6224.59 & 15458.30 & 788 & 982028.63 & 1.26 & -25.01 & -25.51 \\
\hline ML52 & $62 \quad 25.13$ & $154 \quad 59.76$ & 774 & 982030.38 & 1.07 & -24.73 & -25.22 \\
\hline M153 & $62 \quad 25.79$ & $\begin{array}{ll}155 & 0.69\end{array}$ & 752 & 982031.31 & -0.94 & -25.98 & -26.45 \\
\hline M154 & $62 \quad 26.17$ & $155 \quad 2.48$ & 730 & 982031.62 & -3.13 & -27.45 & -27.91 \\
\hline M155 & $62 \quad 27.03$ & 3.09 & 717 & 982033.12 & -3.97 & -27.81 & -28.26 \\
\hline M156 & $\begin{array}{ll}62 & 27.77\end{array}$ & $155 \quad 2.89$ & 716 & 982035.13 & -2.94 & -26.79 & -27.24 \\
\hline$M 157$ & $62 \quad 28.63$ & $155 \quad 2.77$ & 701 & 982037.37 & -3.17 & -26.49 & -26.94 \\
\hline M158 & $62 \quad 29.34$ & $155 \quad 3.23$ & 689 & 982037.37 & -5.15 & -28.10 & -28.53 \\
\hline M159 & 6230.15 & 1553.62 & 673 & 982039.12 & -5.92 & -28.34 & -28.77 \\
\hline M160 & $62 \quad 30.89$ & 3.41 & 651 & 982040.87 & -7.06 & -28.75 & -29.16 \\
\hline $\mathrm{M} 161$ & 6231.72 & $155 \quad 2.80$ & 639 & 982042.81 & -7.39 & -28.70 & -29.11 \\
\hline$M 162$ & $62 \quad 32.44$ & $155 \quad 3.11$ & 622 & 982043.81 & -8.94 & -29.70 & -30.10 \\
\hline $\mathrm{M} 163$ & 6251.21 & 15539.25 & 307 & 982110.69 & 5.26 & -4.90 & -5.10 \\
\hline M164 & 6250.63 & $155 \quad 37.25$ & 305 & 982109.12 & 4.23 & -5.91 & -6.11 \\
\hline M165 & $62 \quad 49.72$ & $155 \quad 37.07$ & 306 & 982105.62 & 1.85 & -8.34 & -8.53 \\
\hline$M 166$ & $62 \quad 49.12$ & $155 \quad 34.93$ & 323 & 982104.81 & 2.42 & -8.01 & -8.20 \\
\hline$M 167$ & 6248.49 & $155 \quad 33.62$ & 320 & 982099.63 & -1.24 & -11.89 & -12.10 \\
\hline$M 168$ & 6248.05 & 15531.96 & 339 & 982097.12 & -1.47 & -12.77 & -12.98 \\
\hline $\mathrm{M} 169$ & 6248.07 & $155 \quad 30.17$ & 340 & 982096.62 & -1.86 & -13.20 & -13.41 \\
\hline M170 & $62 \quad 47.63$ & $155 \quad 27.72$ & 359 & 982097.88 & 1.70 & -10.26 & -10.48 \\
\hline M171 & 6247.07 & 15527.38 & 357 & 982097.88 & 2.20 & -9.70 & -9.92 \\
\hline$M 172$ & 6246.58 & 15525.82 & 361 & 982096.19 & 1.53 & -10.51 & -10.74 \\
\hline$M 173$ & 6245.89 & 15524.56 & 365 & 982093.50 & 0.05 & -12.12 & -12.35 \\
\hline$M 274$ & 6244.72 & $155 \quad 22.64$ & 390 & 982087.12 & -2.53 & $-15,54$ & -15.79 \\
\hline M175 & $62 \quad 43.91$ & 15520.84 & 413 & 982089.87 & 3.46 & -10.31 & -10.57 \\
\hline$M 176$ & 6242.96 & $155 \quad 20.86$ & 408 & 982087.00 & 1.25 & -12.35 & -12.61 \\
\hline M177 & $62 \quad 42.17$ & $155 \quad 20.26$ & 408 & 982087.88 & 3.04 & -10.51 & -10.77 \\
\hline M1 78 & 6240.81 & $155 \quad 19.02$ & 469 & 982073.31 & -4.08 & -19.70 & -20.00 \\
\hline $\mathrm{M} 179$ & 6237.44 & $155 \quad 15.56$ & 521 & 982063.00 & -5.33 & -22.67 & -23.00 \\
\hline M180 & $62 \quad 9.17$ & $154 \quad 19.44$ & 2496 & 981899.69 & 52.23 & -16.19 & -17.50 \\
\hline M181 & $62 \quad 9.90$ & $154 \quad 19.31$ & 2438 & 981903.81 & 49.98 & -20.62 & -21.96 \\
\hline$M 182$ & $62 \quad 10.45$ & $154 \quad 18.91$ & 2388 & 981906.81 & 47.59 & -23.22 & -24.58 \\
\hline$M 183$ & $62 \quad 11.02$ & $154 \quad 18.66$ & 2346 & 981910.19 & 46.29 & -23.63 & -24.97 \\
\hline$M 184$ & 6211.83 & $154 \quad 18.85$ & 2289 & 981913.37 & 43.18 & -24.44 & -25.73 \\
\hline M185 & $62 \quad 12.49$ & $154 \quad 19.34$ & 2227 & 981918.31 & 41.32 & -24.81 & -26.07 \\
\hline M186 & $62 \quad 13.11$ & $154 \quad 19.76$ & 2163 & 981923.31 & 39.55 & -25.30 & -26.54 \\
\hline M187 & $62 \quad 13.47$ & 15420.86 & 2110 & 981926.69 & 37.57 & -24.66 & -25.85 \\
\hline
\end{tabular}


Table 1 (con.)

\begin{tabular}{|c|c|c|c|c|c|c|c|}
\hline at1on & Latitude & Longltude & $\begin{array}{l}\text { Elev } \\
(f t)\end{array}$ & Observed & EAA & $\mathrm{CBA}(2.62)$ & $\mathrm{CBA}(2.6$ \\
\hline$M 188$ & 6213.69 & $154 \quad 22.04$ & 2055 & 981930.13 & 35.52 & -25.37 & -26.53 \\
\hline$M 189$ & 6214.01 & 15423.44 & 1977 & 981935.19 & 32.86 & -25.05 & -26.16 \\
\hline M190 & 6214.03 & 15424.70 & 1934 & 981938.19 & 31.81 & -25.37 & -26.46 \\
\hline$M 191$ & 6214.35 & 15426.00 & 1864 & 981945.50 & 32.13 & -23.80 & -24.86 \\
\hline M192 & $62 \quad 14.82$ & 15427.14 & 1802 & 981952.12 & 32.37 & -23.84 & -24.91 \\
\hline M193 & $\begin{array}{ll}62 & 15.37\end{array}$ & $154 \quad 27.94$ & 1742 & 981958.37 & 32.32 & -23.08 & -24.14 \\
\hline M194 & $62 \quad 16.17$ & 15429.04 & 1693 & 981961.50 & 29.75 & -24.93 & -25.97 \\
\hline M195 & $62 \quad 16.85$ & 15430.04 & 1619 & 981962.31 & 22.69 & -29.84 & -30.84 \\
\hline M196 & $62 \quad 17.69$ & 15430.47 & 1563 & 981966.50 & 20.61 & -29.31 & -30.26 \\
\hline M197 & 6218.44 & 15431.53 & 1515 & 981970.37 & 19.08 & -29.54 & -30.47 \\
\hline M198 & $62 \quad 19.23$ & 15432.62 & 1433 & 981976.50 & 16.50 & -30.02 & -30.91 \\
\hline M199 & 6219.91 & 15433.85 & 1387 & 981982.37 & 17.21 & -28.66 & -29.54 \\
\hline $\mathrm{M} 200$ & $62 \quad 20.67$ & $154 \quad 34.59$ & 1317 & 981988.00 & 15.31 & -28.29 & -29.12 \\
\hline $\mathrm{M} 201$ & 6221.60 & 15434.04 & 1241 & 981994,69 & 13.67 & -27.42 & -28.21 \\
\hline $\mathrm{M} 202$ & 6222.46 & 15433.43 & 1210 & 981998.31 & 13.35 & -25.78 & -27.54 \\
\hline M203 & $62 \quad 23.35$ & $154 \quad 33.03$ & 1155 & 982003.81 & 12.50 & -25.79 & -26.52 \\
\hline M204 & $62 \quad 24.25$ & 15432.61 & 1111 & 982008.19 & 11.69 & -25.14 & -25.84 \\
\hline M205 & $62 \quad 25.04$ & $154 \quad 33.02$ & 1048 & 982014.87 & 11.48 & -23.33 & -24.00 \\
\hline M206 & $62 \quad 25.99$ & $154 \quad 32.47$ & 1000 & 982019.37 & 10.26 & -23.00 & -23.63 \\
\hline M207 & $62 \quad 26.81$ & $154 \quad 32.72$ & 965 & 982024.12 & 10.66 & -21.45 & -22.06 \\
\hline M208 & $62 \quad 27.63$ & $154 \quad 32.36$ & 924 & 982028.50 & 10.19 & -20.58 & -21.17 \\
\hline M209 & $62 \quad 28.47$ & $154 \quad 32.41$ & 886 & 982033.50 & 10.57 & -18.93 & -19.49 \\
\hline M210 & $62 \quad 29.24$ & $154 \quad 31.88$ & 846 & 982039.12 & 11.46 & -16.71 & -17.24 \\
\hline$M 211$ & $62 \quad 30.22$ & $154 \quad 32.09$ & 811 & 982045.31 & 13.18 & -13.85 & -14.37 \\
\hline$M 212$ & 6231.06 & 15431.68 & 782 & 982048.37 & 12.56 & -13.53 & -14.03 \\
\hline M213 & 6231.99 & 15431.93 & 748 & 982050.81 & 10.58 & -14.36 & -14.84 \\
\hline$M 214$ & $62 \quad 32.76$ & $154 \quad 32.81$ & 714 & 982054.50 & 10.12 & -13.69 & -14.15 \\
\hline$M 215$ & 6233.67 & $154 \quad 33,46$ & 674 & 982057.31 & 8.00 & -14.47 & -14.90 \\
\hline M216 & $62 \quad 33.45$ & 15438.42 & 689 & 982052.81 & 5.13 & -17.82 & -18.25 \\
\hline$M 217$ & 6230.96 & $154 \quad 40.68$ & 719 & 982047.50 & 5.79 & -17.19 & -17.63 \\
\hline$M 218$ & $62 \quad 30.57$ & $154 \quad 43.03$ & 2490 & 981931.31 & 56.63 & -15.69 & -17.07 \\
\hline$M 219$ & 6230.18 & $154 \quad 45.46$ & 1291 & 982017.00 & 30.08 & -12.14 & -12.95 \\
\hline M220 & $62 \quad 28.64$ & $154 \quad 48.96$ & 1451 & 982003.62 & 33.60 & -12.86 & -13.74 \\
\hline$M 221$ & $62 \quad 28.24$ & 15452.12 & 1788 & 981976.00 & 38.18 & -17.19 & -18.24 \\
\hline M222 & $62 \quad 26.86$ & 15455.43 & 948 & 982020.50 & 5.40 & -26.13 & -26.73 \\
\hline$M 223$ & $62 \quad 23.43$ & $155 \quad 8.32$ & 1053 & 981999.81 & -1.14 & -36.11 & -36.78 \\
\hline M224 & 6221.45 & 15515.44 & 800 & 982021.50 & -0.79 & -27.48 & -27.99 \\
\hline$M 225$ & 6220.01 & 15521.29 & 641 & 982029.31 & -6.24 & -27.37 & -27.77 \\
\hline M226 & $62 \quad 18.04$ & $155 \quad 27.72$ & 1860 & 981944.50 & 26.14 & -32.79 & -33.91 \\
\hline$M 227$ & 6159.49 & 15436.58 & 1378 & 981952.87 & 12.37 & -33.02 & -33.88 \\
\hline$M 228$ & 6158.64 & 15436.65 & 1419 & 981947.62 & 11.96 & -34.82 & -35.71 \\
\hline M229 & 6157.81 & 15436.49 & 1442 & 981943.69 & 11.32 & -36.15 & -37.06 \\
\hline$M 230$ & $61 \quad 57.09$ & $154 \quad 35.51$ & 1463 & 981940.31 & 10.74 & -37.42 & -38.34 \\
\hline M231 & $61 \quad 56.39$ & $154 \quad 34.52$ & 1477 & 981938.88 & 11.65 & -37.01 & -37.94 \\
\hline M232 & $61 \quad 55.59$ & $154 \quad 33.77$ & 1502 & 981935.38 & 11.44 & -38.04 & -38.98 \\
\hline$M 233$ & 6155.25 & 15432.12 & 1528 & 981928.87 & 7.87 & -42.55 & -43.51 \\
\hline M234 & 6155.00 & 15430.34 & 1564 & 981924.00 & 6.63 & -44.89 & -45.87 \\
\hline
\end{tabular}


Table 1 (con.)

\begin{tabular}{|c|c|c|c|c|c|c|c|c|}
\hline at1on & Lat1tude & Long & gitude & $\begin{array}{l}\text { Elev } \\
(\mathrm{f} t)\end{array}$ & Observed & FAA & $\mathrm{CBA}(2.62)$ & $\mathrm{CBA}(2.6$ \\
\hline M235 & 6154.78 & 154 & 28.64 & 1608 & 981915.00 & 2.07 & -50.72 & -51.73 \\
\hline M236 & 6154.33 & 154 & 27.01 & 1629 & 981912.19 & 1.78 & -51.41 & -52.43 \\
\hline M237 & 6153.92 & 154 & 25.40 & 1653 & 981915.19 & 7.53 & -45.99 & -47.02 \\
\hline M238 & 6153.46 & 154 & 23.83 & 1695 & 981912.37 & 9.34 & -45.07 & -46.11 \\
\hline$M 239$ & 6152.89 & 154 & 22.42 & 1729 & 981908.81 & 9.61 & -45.56 & -46.62 \\
\hline$M 240$ & 6152.38 & 154 & 20.93 & 1770 & 981902.62 & 7.92 & -47.95 & -49.02 \\
\hline M241 & 6151.83 & 154 & 19.47 & 1810 & 981896.81 & 6.54 & -49.10 & -50.16 \\
\hline$M 242$ & 6151.36 & 154 & 17.99 & 1852 & 981890.31 & 4.63 & -51.37 & -52.44 \\
\hline $\mathrm{M} 243$ & 6150.92 & 154 & 16.37 & 1887 & 981885.81 & 3.96 & -53.02 & $-54 \cdot 10$ \\
\hline$M 244$ & 6150.30 & 154 & 15.01 & 1930 & 981878.81 & 1.80 & -52.76 & -53.80 \\
\hline$M 245$ & 6149.86 & 154 & 14.43 & 1950 & 981874.87 & 0.29 & -50.15 & -51.11 \\
\hline M246 & 6148.81 & 154 & 13.05 & 2044 & 981858.62 & -5.88 & -51.93 & -52.81 \\
\hline M247 & 6148.12 & 154 & 12.04 & 2135 & 981844.37 & -10.65 & -57.96 & -58.87 \\
\hline M248 & 6142.25 & 154 & 6.95 & 4403 & 981698.12 & 63.77 & -36.63 & -38.55 \\
\hline M249 & 6136.07 & 154 & 2.95 & 1990 & 981837.19 & -16.24 & -63.91 & -64.82 \\
\hline$M 250$ & $62 \quad 6.36$ & 154 & 9.87 & 2711 & 981878.87 & 55.13 & -31.73 & -33.39 \\
\hline M251 & $62 \quad 12.14$ & 154 & 9.07 & 2291 & 981912.12 & 41.68 & -30.66 & -32.05 \\
\hline M252 & $62 \quad 16.49$ & 154 & 3.23 & 1964 & 981932.69 & 26.04 & -33.74 & -34.88 \\
\hline M253 & $62 \quad 30.97$ & 153 & 43.93 & 1668 & 981982.37 & 29.91 & -24.90 & -25.94 \\
\hline$M 254$ & 6259.54 & 153 & 0.11 & 1227 & 982042.62 & 13.52 & -27.26 & -28.04 \\
\hline M255 & 1.10 & 153 & 9.22 & 1078 & 982057.31 & 12.25 & -23.65 & -24.33 \\
\hline M256 & 6253.31 & 153 & 13.00 & 1363 & 982017.81 & 9.11 & -36.39 & -37.26 \\
\hline M257 & 6250.80 & 153 & 27.63 & 988 & 982043.81 & 2.94 & -29.99 & -30.62 \\
\hline M258 & 6249.18 & 153 & 34.69 & 944 & 982049.12 & 6.07 & -25.42 & -26.02 \\
\hline$M 259$ & 6246.67 & 153 & 42.85 & 830 & 982072.87 & 22.29 & -5.38 & -5.91 \\
\hline$M 260$ & 6244.99 & 153 & 53.71 & 753 & 982066.62 & 10.79 & -14.34 & -14.81 \\
\hline M261 & $62 \quad 44.28$ & 153 & 50.69 & 769 & 982079.13 & 25.58 & -0.03 & -0.52 \\
\hline M262 & 6241.15 & 154 & 4.75 & 910 & 982044.87 & 8.56 & -21.68 & -22.25 \\
\hline$M 263$ & 6238.64 & 154 & 14.05 & 82 & .12 & 22.12 & -5.52 & -6.05 \\
\hline M264 & $62 \quad 28.01$ & 154 & 1.46 & 1529 & 981990.62 & 28.71 & -21.31 & -22.27 \\
\hline$M 300$ & $62 \quad 23.49$ & 153 & 7.82 & 290 & 981863.19 & 35.89 & -53.64 & -55.36 \\
\hline $\mathrm{M} 301$ & $62 \quad 23.04$ & 153 & 10.21 & 2740 & 981877.37 & 35.57 & -50.50 & -52.14 \\
\hline M302 & $62 \quad 23.15$ & 153 & 12.61 & 2585 & 981887.00 & 30.46 & -48.93 & -50.44 \\
\hline $\mathrm{M} 303$ & 6224.31 & 153 & 14.53 & 2405 & 981892.31 & 17.42 & -56.08 & -57.48 \\
\hline$M 304$ & $62 \quad 25.25$ & 153 & 16.37 & 229 & 981905.31 & 19.21 & -46.59 & -47.85 \\
\hline M305 & $62 \quad 26.42$ & 153 & 17.20 & 2140 & 981915.87 & 13.46 & -46.63 & -47.77 \\
\hline M306 & $62 \quad 27.66$ & 153 & 19.55 & 2011 & 981933.12 & 16.93 & -40.78 & -41.88 \\
\hline M307 & $62 \quad 28.29$ & 153 & 22.37 & 1833 & 981949.87 & 16.25 & -38.20 & -39.24 \\
\hline M308 & 6229.55 & 153 & 24.74 & 1651 & 981964.69 & 12.37 & -38.33 & -39.30 \\
\hline M309 & 6231.05 & 153 & 24.84 & 1502 & 981982,12 & 13.95 & -32.54 & -33.43 \\
\hline M310 & $\begin{array}{ll}62 & 32.87\end{array}$ & 153 & 25.75 & 1288 & 982001.12 & 10.50 & -30.87 & -31.66 \\
\hline M311 & 6234.18 & 153 & 27.98 & 1138 & 982013.87 & 7.66 & -29.59 & -30.30 \\
\hline M312 & $62 \quad 36.34$ & 153 & 29.62 & 1152 & 982021.63 & 13.95 & -23.94 & -24.67 \\
\hline M313 & $62 \quad 36.54$ & 153 & 24.04 & 1331 & 982009.81 & 18.68 & -23.91 & -24.72 \\
\hline M314 & $62 \quad 36.64$ & 153 & 19.08 & 1489 & 982001.00 & 24.68 & -23.44 & -24.36 \\
\hline$M 315$ & 6235.44 & 153 & 15.83 & 1626 & 981990.87 & 28.92 & -23.23 & -24.23 \\
\hline M 316 & $62 \quad 33.57$ & 153 & 14.32 & 1737 & 981972.38 & 23.25 & -29.16 & -30.16 \\
\hline
\end{tabular}


Table 1 (con.)

\begin{tabular}{|c|c|c|c|c|c|c|c|c|}
\hline Station & Lat1tude & Long & 1tude & $\begin{array}{l}E 1 e v, \\
(E t)^{a}\end{array}$ & Observed & FAA & $\mathrm{CBA}(2.62)$ & $\mathrm{CBA}(2$. \\
\hline$M 317$ & 6231.74 & 153 & 9.69 & 1953 & 981943.50 & 16.86 & -37.33 & -38.36 \\
\hline$M 318$ & 6231.42 & 153 & 4.77 & 2145 & 981930.69 & 22.51 & -38.58 & -39.74 \\
\hline M319 & $62 \quad 32.57$ & 153 & 0.49 & 2394 & 981919.31 & 33.11 & -37.66 & -39.01 \\
\hline $\mathrm{M} 320$ & $62 \quad 32.96$ & 152 & 57.49 & 2530 & 981908.87 & 35.12 & -38.76 & -40.17 \\
\hline M321 & 6233.85 & 152 & 53.98 & 2708 & 981902.37 & 44.14 & -34.95 & -36.46 \\
\hline $\mathrm{M} 324$ & 6239.67 & 153 & 8.11 & 2121 & 981962.87 & 42.24 & -25.23 & -26.52 \\
\hline$M 325$ & $62 \quad 39.82$ & 153 & 5.93 & 2264 & 981956.62 & 49.21 & -23.49 & -24.87 \\
\hline M326 & 6240.05 & 153 & 3.80 & 2343 & 981953.12 & 52.84 & -23.49 & -24.95 \\
\hline M327 & 6238.35 & 153 & 0.42 & 2858 & 981929.69 & 80.01 & -11.65 & -13.40 \\
\hline$M 328$ & 6237.52 & 152 & 58.90 & 3121 & 981906.81 & 82.93 & -15.91 & -17.80 \\
\hline M329 & 6236.67 & 152 & 59.23 & 3426 & 981875.31 & 81.07 & -22.60 & -24.58 \\
\hline M330 & $62 \quad 38.59$ & 153 & 9.71 & 3558 & 981873.19 & 89.07 & -22.80 & -24.94 \\
\hline M331 & 6238.71 & 153 & 12.85 & 2683 & 981927.12 & 60.59 & -25.66 & -27.31 \\
\hline M332 & 6237.11 & 153 & 13.58 & 2716 & 981933.63 & 72.17 & -15.33 & -17.00 \\
\hline M333 & 6235.16 & 153 & 20.57 & 2116 & 981950.12 & 44.55 & -24.23 & -25.55 \\
\hline M334 & 6240.45 & 153 & 10.01 & 2028 & 981970.81 & 40.49 & -25.56 & -26.82 \\
\hline M335 & 6241.14 & 153 & 11.90 & 1878 & 981975.31 & 30.04 & -31.92 & -33.11 \\
\hline M336 & 6241.59 & 153 & 13.54 & 1791 & 981981.50 & 27.48 & -31.83 & -32.96 \\
\hline M337 & 6241.73 & 153 & 15.50 & 1734 & 981984.87 & 25.35 & -32.19 & -33.29 \\
\hline M338 & $62 \quad 41.79$ & 153 & 18.06 & 1589 & 981996.38 & 23.16 & -29.61 & -30.62 \\
\hline M339 & 6242.01 & 153 & 20.71 & 1454 & 982006.69 & 20.49 & -27.84 & -28.77 \\
\hline M340 & 6242.58 & 153 & 23.15 & 1359 & 982010.12 & 14.20 & -31.03 & -31.90 \\
\hline M34L & 6243.29 & 153 & 24.56 & 1274 & 982011.50 & 6.73 & -35.66 & -36.47 \\
\hline M342 & 6244.02 & 153 & 25.55 & 1218 & 982012.12 & 1.16 & -39.37 & -40.14 \\
\hline$M 343$ & 6245.09 & 153 & 26.36 & 1148 & 982014.31 & -4.47 & -42.75 & -43.48 \\
\hline$M 344$ & 6246.23 & 153 & 27.98 & 1125 & 982015.87 & -6.45 & -43.98 & -44.69 \\
\hline M345 & $62 \quad 47.65$ & 153 & 30.41 & 969 & 981970.87 & -67.88 & -100.21 & -100.83 \\
\hline M346 & $62 \quad 48.48$ & 153 & 32.69 & 921 & 982045.31 & 0.99 & -29.74 & -30.33 \\
\hline$M 347$ & 6249.73 & 153 & 36.54 & 833 & 982060.81 & 6.70 & -21.08 & -21.61 \\
\hline M348 & 6250.39 & 153 & 38.48 & 785 & 982066.00 & 6.55 & -19.65 & -20.16 \\
\hline M349 & $62 \quad 43.77$ & 152 & 56.60 & 2269 & 981952.87 & 41.16 & -32.50 & -33.91 \\
\hline M350 & $62 \quad 44.78$ & 152 & 58.88 & 2122 & 981951.00 & 24.21 & -45.49 & -46.82 \\
\hline M351 & 6245.51 & 153 & 0.20 & 2018 & 981952.31 & 14.79 & -52.02 & -53.29 \\
\hline M352 & 6247.08 & 153 & 0.83 & 1932 & 981968.37 & 20.90 & -43.26 & -44.48 \\
\hline M353 & 6248.36 & 153 & 2.33 & 1786 & 981977.81 & 14.92 & -44.53 & -45.67 \\
\hline$M 354$ & 6249.38 & 153 & 4.28 & 1634 & 981991.19 & 12.81 & -41.61 & -42.65 \\
\hline M355 & 6250.35 & 153 & 6.45 & 1524 & 982002.37 & 12.48 & -38.29 & -39.26 \\
\hline M356 & 6251.55 & 153 & 8.59 & 1400 & 982013.19 & 10.12 & -36.55 & -37.44 \\
\hline M357 & 6252.43 & 153 & 10.76 & 1286 & 982021.81 & 6.94 & -35.93 & -36.75 \\
\hline M358 & $62 \quad 53.39$ & 153 & 13.09 & 1187 & 982030.87 & 5.53 & -34.08 & -34.84 \\
\hline M359 & 6254.08 & 153 & 14.99 & 1103 & 982039.37 & 5.30 & -31.51 & -32.21 \\
\hline M360 & 6254.92 & 153 & 17.25 & 1032 & 982045.81 & 4.02 & -30.43 & -31.08 \\
\hline M361 & 6255.51 & 153 & 19.81 & 979 & 982051.12 & 3.64 & -29.04 & -29.66 \\
\hline$M 362$ & 6255.15 & 153 & 25.54 & 879 & 982058.62 & 2.17 & -27.16 & -27.72 \\
\hline M363 & 6255.17 & 153 & 29.43 & 806 & 982066.31 & 2.92 & -24.00 & -24.51 \\
\hline M364 & 6255.96 & 153 & 33.85 & 762 & 982070.81 & 2.36 & -23.10 & -23.59 \\
\hline M365 & $63 \quad 23.24$ & 154 & 21.55 & 1163 & 982089.19 & 25.22 & -9.91 & -10.58 \\
\hline
\end{tabular}


Table 1 (con.)

\begin{tabular}{|c|c|c|c|c|c|c|c|}
\hline ten & Latitude & Longttude & $\begin{array}{l}\text { Elev } \\
(E t)\end{array}$ & Observed & FAA & $\mathrm{CBA}(2.62)$ & $\mathrm{CBA}(2.67)$ \\
\hline M366 & 6251.12 & $152 \quad 25.73$ & 1842 & 981982.81 & 21.79 & -37.85 & -38.98 \\
\hline M367 & 6251.02 & $152 \quad 27.29$ & 1829 & 981984.63 & 22.50 & -36.94 & -38.07 \\
\hline M368 & 6251.19 & $152 \quad 28.54$ & 1805 & 981988.31 & 23.81 & -35.16 & -36.29 \\
\hline M369 & 6251.57 & 15231.15 & 1763 & 981994.12 & 25.17 & -32.68 & -33.79 \\
\hline $\mathrm{M} 370$ & 6251.30 & $152 \quad 29.57$ & 1785 & 981990.87 & 24.41 & -34.03 & -35.15 \\
\hline$M 371$ & 6251.61 & 15232.65 & 1736 & 981994.12 & 22.56 & -34.50 & -35.59 \\
\hline$M 372$ & 6252.07 & 15233.85 & 1723 & 981997.87 & 24.65 & -32.06 & -33.14 \\
\hline $\mathrm{M} 373$ & $62 \quad 52.59$ & 15234.74 & 1710 & 981998.13 & 22.97 & -33.40 & -34.48 \\
\hline M374 & 6253.04 & $152 \quad 35.80$ & 1695 & 981996.62 & 19.48 & -36.24 & -37.31 \\
\hline M375 & $62 \quad 52.84$ & $152 \quad 37.14$ & 1672 & 981996.31 & 17.27 & -37.89 & -38.95 \\
\hline M976 & 6252.91 & 15238.54 & 1661 & 981997.12 & 16.89 & -37.91 & -38.96 \\
\hline M377 & 6253.05 & 15239.94 & 1644 & 981996.50 & 14.53 & $-39.7 \mathrm{l}$ & -40.75 \\
\hline M378 & $62 \quad 53.36$ & 15241.57 & 1625 & 981996.62 & 12.48 & -40.95 & -41.97 \\
\hline M379 & $62 \quad 53.44$ & 15242.89 & 1609 & 981998.13 & 12.34 & -40.87 & -41.89 \\
\hline M380 & $62 \quad 53.49$ & 15244.38 & 1577 & 982000.87 & 12.10 & -39.96 & -40.95 \\
\hline M381 & $62 \quad 53.70$ & 15246.07 & 1553 & 981999.69 & 8.37 & -42.74 & -43.71 \\
\hline M382 & 6253.95 & 15247.86 & 1520 & 982002.19 & 7.47 & -42.76 & -43.72 \\
\hline M383 & 6254.21 & 15249.40 & 1490 & 982003.81 & 5.89 & -43.47 & -44.41 \\
\hline M384 & 6254.53 & $152 \quad 50.83$ & 1466 & 982008.19 & 7.67 & -40.89 & -41.81 \\
\hline M385 & 6255.06 & $152 \quad 52.00$ & 1439 & 982013.38 & 9.72 & -37.92 & -38.83 \\
\hline$M 386$ & $62 \quad 55.52$ & $152 \quad 53.62$ & 1411 & 982015.81 & 8.89 & -38.07 & -38.97 \\
\hline M387 & 6256.14 & 15254.85 & 1371 & 982026.19 & 14.81 & -30.82 & -31.70 \\
\hline M388 & 6256.71 & $152 \quad 56.22$ & 1348 & 982023.38 & 9.15 & -35.82 & -36.68 \\
\hline 1389 & $62 \quad 57.38$ & 15256.98 & 1317 & 982027.31 & 9.28 & -34.65 & -35.49 \\
\hline$M 390$ & $62 \quad 57.89$ & 15257.91 & 1293 & 982030.19 & 9.30 & -33.85 & -34.68 \\
\hline M391 & $62 \quad 58.37$ & 15258.72 & 1270 & 982033.87 & 10.32 & -32.07 & -32.88 \\
\hline $\mathrm{M} 392$ & 6258.98 & 15259.32 & 1252 & 982038.00 & 11.89 & -29.85 & -30.65 \\
\hline M393 & $62 \quad 59.58$ & $152 \quad 59.90$ & 1225 & 982042.81 & 13.39 & -27.35 & -28.13 \\
\hline$M 394$ & $\begin{array}{ll}63 & 0.12\end{array}$ & 1530.95 & 1209 & 982043.81 & 12.22 & -28.00 & -28.77 \\
\hline M395 & $\begin{array}{ll}63 & 0.46\end{array}$ & 1532.14 & 1180 & 982045.37 & 10.80 & -28.49 & -29.24 \\
\hline$M 396$ & $\begin{array}{ll}63 & 0.78\end{array}$ & $153 \quad 2.91$ & 1162 & 982047.31 & 10.55 & -28.15 & -28.89 \\
\hline M397 & $\begin{array}{ll}63 & 1.02\end{array}$ & 4.06 & 1139 & 982049.87 & 10.68 & -27.25 & -27.97 \\
\hline M398 & $63 \quad 1.29$ & 15312.97 & 1017 & 982060.62 & 9.57 & -24.17 & -24.81 \\
\hline$M 399$ & $63 \quad 1.02$ & $153 \quad 11.82$ & 1032 & 982058.12 & 8.86 & -25.54 & -26.19 \\
\hline M400 & $\begin{array}{ll}63 & 0.95\end{array}$ & 15310.22 & 1053 & 982056.81 & 9.61 & -25.40 & -26.07 \\
\hline $\mathrm{M401}$ & $63 \quad 1.15$ & 1538.55 & 1086 & 982056.62 & 12.25 & -23.85 & -24.54 \\
\hline$M 402$ & $\begin{array}{ll}63 & 0.90\end{array}$ & 1536.58 & 1102 & 982052.69 & 10.14 & -26.58 & -27.28 \\
\hline M403 & 631.10 & 5.15 & 1117 & 982054.19 & 12.82 & -24.37 & -25.08 \\
\hline M404 & 631.15 & $153 \quad 14.26$ & 989 & 982060.62 & 7.12 & -25.87 & -26.50 \\
\hline M405 & $63 \quad 1.81$ & 15315.40 & 980 & 982062.81 & 7.67 & -25.02 & -25.65 \\
\hline$M 406$ & $63 \quad 2.46$ & 15316.14 & 953 & 982065.12 & 6.67 & -25.13 & -25.74 \\
\hline$M 407$ & $63 \quad 2.67$ & $153 \quad 17.99$ & 924 & 982067.12 & 5.64 & -25.21 & -25.79 \\
\hline$M 408$ & $63 \cdot 3.09$ & $153 \quad 19.30$ & 908 & 982069.69 & 6.20 & -24.12 & -24.70 \\
\hline$M 409$ & $63 \quad 3.28$ & 15320.99 & 890 & 982071.37 & 5.99 & -23.74 & -24.31 \\
\hline M410 & $63 \quad 3.94$ & 15321.86 & 870 & 982072.63 & 4.47 & -24.59 & -25.14 \\
\hline M411 & 634.10 & $153 \quad 23.35$ & 858 & 982075.31 & 5.90 & -22.75 & -23.30 \\
\hline$M 412$ & 634.69 & 15324.60 & 834 & 982076.81 & 4.45 & -23.41 & -23.94 \\
\hline
\end{tabular}


Table 1 (con.)

\begin{tabular}{|c|c|c|c|c|c|c|c|c|c|}
\hline \multirow{2}{*}{$\begin{array}{c}\text { Station } \\
M 413\end{array}$} & \multicolumn{2}{|c|}{ Lat1tude } & \multicolumn{2}{|c|}{ Long1tude } & $\begin{array}{l}\text { Elev }_{\dot{a}} \\
(\mathrm{ft})^{\mathrm{a}}\end{array}$ & Observed & FAA & $\mathrm{CBA}(2.62)$ & $\operatorname{CBA}(2.67)$ \\
\hline & 63 & 4.99 & 153 & 26.72 & 806 & 982079.13 & 3.72 & -23.21 & -23.73 \\
\hline M414 & 63 & 5.17 & 153 & 27.83 & 787 & 982082.87 & 5.53 & -20.76 & -21.27 \\
\hline M415 & 62 & 58.12 & 151 & 58.26 & 2598 & 981930.56 & 32.15 & -50.63 & -52.20 \\
\hline$M 416$ & 62 & 58.52 & 151 & 59.86 & 2533 & 981934.50 & 29.47 & -52.04 & -53.59 \\
\hline M417 & 62 & 58.92 & 152 & 1.74 & 2450 & 981939.69 & 26.35 & -53.34 & -54.86 \\
\hline M418 & 62 & 59.40 & 152 & 3.42 & 2415 & 981943.63 & 26.41 & -52.61 & -54.12 \\
\hline M419 & 62 & 59.32 & 152 & 5.18 & 2348 & 981950.63 & 27.24 & -49.03 & -50.48 \\
\hline$M 420$ & 62 & 59.14 & 152 & 6.43 & 2306 & 981954.69 & 27.57 & -47.41 & -48.84 \\
\hline M421 & 62 & 59.24 & 152 & 8.45 & 2253 & 981959.62 & 27.34 & -46.58 & -47.99 \\
\hline M422 & 62 & 59.16 & 152 & 10.19 & 2202 & 981964.37 & 27.41 & -45.00 & -46.38 \\
\hline M423 & 62 & 59.32 & 152 & L L .84 & 2159 & 981969.38 & 28.21 & -43.09 & -44.45 \\
\hline M424 & 62 & 59.16 & 152 & 13.61 & 2111 & 981972.87 & 27.41 & -42.40 & -43.73 \\
\hline M425 & 62 & 59.22 & 152 & 15.29 & 2061 & 981976.87 & 26.62 & -41.54 & -42.84 \\
\hline M426 & 62 & 59.34 & 152 & 17.14 & 2011 & 981981.81 & 26.61 & -40.14 & -41.42 \\
\hline M427 & 63 & 0.02 & 152 & 17.99 & 1955 & 981987.37 & 26.12 & -38.85 & -40.09 \\
\hline M428 & 63 & 0.77 & 152 & 18.58 & 1912 & 981991.81 & 25.63 & -37.95 & -39.17 \\
\hline M429 & 63 & 1.58 & 152 & 18.73 & 1858 & 981996.87 & 24.64 & $-37,19$ & -38.37 \\
\hline M430 & 63 & 2.26 & 152 & 19.13 & 1806 & 982002.62 & 24.62 & -35.51 & -36.66 \\
\hline M431 & 63 & 3.14 & 152 & 19.50 & 1760 & 982005.69 & 22.28 & -36.34 & -37.46 \\
\hline$M 432$ & 63 & 3.94 & 152 & 20.08 & 1724 & 982007.87 & 20.19 & -37.27 & -38.36 \\
\hline M433 & 63 & 4.76 & 152 & 20.16 & 1666 & 982013.12 & 18.90 & -36.64 & -37.70 \\
\hline$M 434$ & 63 & 5.59 & 152 & 20.60 & 1638 & 982017.87 & 20.02 & -34.67 & -35.71 \\
\hline M435 & 63 & 6.30 & 152 & 21.01 & 1608 & 982022.19 & 20.68 & -33.03 & -34.05 \\
\hline M436 & 63 & 6.75 & 152 & 21.98 & 1567 & 982028.37 & 22.47 & -29.87 & -30.86 \\
\hline M437 & 63 & 7.54 & 152 & 22.94 & 1539 & 982031.62 & 22.11 & -29.29 & -30.28 \\
\hline$M 438$ & 63 & 7.95 & 152 & 24.21 & 1508 & 982036.19 & 23.26 & -27.11 & -28.07 \\
\hline M439 & 63 & 8.57 & 152 & 25.39 & 1483 & 982036.37 & 20.36 & -29.17 & -30.12 \\
\hline$M 440$ & 63 & 9.00 & 152 & 27.11 & 1447 & 982039.12 & 19.14 & -29.19 & -30.11 \\
\hline M441 & 63 & 9.40 & 152 & 28.93 & 1419 & 982042.69 & 19.59 & -27.81 & -28.72 \\
\hline$M 442$ & 63 & 10.19 & 152 & 29.81 & 1382 & 982047.81 & 20.28 & -25.88 & -26.76 \\
\hline$M 443$ & 63 & 10.83 & 152 & 31.32 & 1343 & 982050.62 & 18.66 & -26.20 & -27.06 \\
\hline M444 & 63 & 11.62 & 152 & 32.17 & 1310 & 982051.62 & 15.53 & -28.23 & -29.06 \\
\hline M445 & 63 & 12.49 & 152 & 33.17 & 1282 & 982051.81 & 12.00 & -30.82 & -31.64 \\
\hline M446 & 63 & 13.27 & 152 & 33.68 & 1246 & 982052.50 & 8.41 & -33.21 & $\sim 34.00$ \\
\hline$M 447$ & 63 & 14.02 & 152 & 34.75 & 1214 & 982057.31 & 9.25 & -31.31 & -32.08 \\
\hline M448 & 63 & 14.78 & 152 & 36.07 & 1182 & 982061.50 & 9.56 & -29.92 & -30.68 \\
\hline M449 & 63 & 15.35 & 152 & 37.76 & 1150 & 982064.37 & 8.75 & -29.67 & -30.40 \\
\hline$M 450$ & 63 & 15.92 & 152 & 39.20 & 1114 & 982067.38 & 7.70 & -29.51 & -30.22 \\
\hline M451 & 63 & 16.77 & 152 & 39.62 & 1090 & 982071.81 & 8.71 & -27.70 & -28.40 \\
\hline M452 & 63 & 17.42 & 152 & 40.47 & 1065 & 982076.37 & 10.23 & -25.35 & -26.03 \\
\hline M453 & 63 & 18.00 & 152 & 41.49 & 1039 & 982076.69 & 7.36 & -27.36 & -28.02 \\
\hline M454 & 63 & 18.62 & 152 & 42.76 & 1008 & 982079.62 & 6.67 & -27.00 & -27.65 \\
\hline M455 & 63 & 19.09 & 152 & 44.25 & 979 & 982083.37 & 7.13 & -25.58 & -26.20 \\
\hline M456 & 63 & 19.51 & 152 & 45.58 & 960 & 982083.00 & 4.42 & -27.66 & -28.27 \\
\hline M457 & 63 & 19.92 & 152 & 47.44 & 928 & 982083.50 & 1.44 & -29.56 & -30.15 \\
\hline$M 458$ & 63 & 19.91 & 152 & 49.19 & 904 & 982084.81 & 0.49 & -29.68 & -30.26 \\
\hline M459 & 63 & 20.15 & 152 & 50.69 & 884 & 982085.19 & -1.34 & -30.86 & -31.42 \\
\hline
\end{tabular}


Table 1 (con.)

\begin{tabular}{|c|c|c|c|c|c|c|c|}
\hline Stat1on & Lat1tude & Longitude & $\begin{array}{l}\text { Elev } \dot{a} \\
(f t)\end{array}$ & Observed & FAA & $\operatorname{CBA}(2.62)$ & $\mathrm{CBA}(2.67$ \\
\hline$M 460$ & $63 \quad 20.49$ & 15252.77 & 864 & 982086.62 & -2.23 & -31.09 & -31.65 \\
\hline M461 & 6320.70 & 15254.37 & 839 & 982088.12 & -3.29 & -31.32 & -31.85 \\
\hline M462 & $63 \quad 20.91$ & $152 \quad 56.27$ & 822 & 982089.00 & -4.25 & -31.71 & -32.24 \\
\hline M463 & 6321.30 & $152 \quad 57.86$ & 806 & 982092.87 & -2.32 & -29.24 & -29.76 \\
\hline M464 & 6321.44 & 15259.56 & 789 & 982097.12 & 0.06 & -26.29 & -26.79 \\
\hline M465 & 6321.50 & $\begin{array}{ll}153 & 1.57\end{array}$ & 775 & 982098.81 & 0.36 & -25.54 & -26.03 \\
\hline M466 & 6321.82 & 3.38 & 760 & 982097.81 & -2.34 & -27.74 & -28.22 \\
\hline M467 & $63 \quad 5.96$ & 15328.73 & 752 & 982084.81 & 3.15 & -21.98 & -22.46 \\
\hline M468 & $63 \quad 6.52$ & 15330.14 & 727 & 982086.50 & 1.79 & -22.50 & -22.96 \\
\hline$M 469$ & 7.16 & 15331.30 & 710 & 982092.50 & 5.43 & -18.30 & -18.75 \\
\hline M470 & $63 \quad 7.74$ & $153 \quad 32.74$ & 691 & 982095.62 & 6.03 & -17.03 & -17.47 \\
\hline$M 471$ & $\begin{array}{ll}63 & 8.57\end{array}$ & $153 \quad 32.65$ & 672 & 982097.81 & 5.42 & -17.00 & -17.42 \\
\hline$M 472$ & 639.25 & $153 \quad 32.49$ & 660 & 982100.87 & 6.61 & -15.38 & -15.80 \\
\hline M473 & 6310.15 & 15332.51 & 642 & 982101.81 & 4.68 & -16.75 & -17.15 \\
\hline M474 & 6310.96 & 15332.30 & 633 & 982104.37 & 5.46 & -15.65 & -16.05 \\
\hline M475 & 6311.20 & 15333.94 & 624 & 982104.81 & 4.73 & -16.11 & -16.50 \\
\hline M476 & $63 \angle 1.30$ & 15335.84 & 613 & 982102.87 & 1.64 & -18.83 & -19.22 \\
\hline M477 & $\begin{array}{ll}63 & 12.01\end{array}$ & 15336.88 & 603 & 982104.12 & I. 12 & -29.02 & -19.40 \\
\hline$M 478$ & 6312.35 & 15338.28 & 589 & 982108.37 & 3.63 & -16.03 & -16.41 \\
\hline M479 & 6313.08 & 15339.20 & 583 & 982113.19 & 7.00 & -12.47 & -12.84 \\
\hline M480 & $\begin{array}{ll}63 & 13.78\end{array}$ & 15340.35 & 568 & 982106.12 & -2.37 & -21.34 & -21.70 \\
\hline M481 & 6314.66 & 15341.81 & 571 & 982107.31 & -1.98 & -21.04 & -21.40 \\
\hline$M 482$ & 6315.53 & 15341.36 & 563 & 982110.37 & -0.68 & -19.47 & -19.83 \\
\hline M483 & 6316.27 & 15341.39 & 571 & 982111.31 & 0.07 & -18.98 & -19.35 \\
\hline M484 & 6316.86 & $153 \quad 39.97$ & 571 & 982109.00 & -2.93 & -22.00 & -22.36 \\
\hline 2485 & $63 \quad 17.37$ & $153 \quad 38.42$ & 568 & 982108.37 & -4.43 & -23.41 & -23.77 \\
\hline M486 & 6317.63 & $153 \quad 36.37$ & 572 & 982106.63 & -6.16 & -25.27 & -25.64 \\
\hline M487 & $63 \quad 18.07$ & 15335.14 & 574 & 982106.81 & -6.32 & -25.50 & -25.86 \\
\hline$M 488$ & $63 \quad 18.71$ & $153 \quad 33.84$ & 584 & 982106.81 & -6.12 & -25.63 & -26.01 \\
\hline M489 & $63 \quad 19.23$ & 15332.62 & 588 & 982107.37 & -5.80 & -25.45 & -25.82 \\
\hline M490 & 6320.27 & 15331.85 & 592 & 982109.37 & -4.75 & -24.53 & -24.91 \\
\hline M491 & $63 \quad 20.92$ & 15330.20 & 599 & 982110.50 & -3.75 & -23.77 & -24.15 \\
\hline M492 & 6321.19 & 15326.20 & 617 & 982112.62 & -0.32 & -20.94 & -21.34 \\
\hline M493 & 6322.30 & 15325.24 & 619 & 982112.00 & -2.05 & -22.73 & -23.13 \\
\hline M494 & $\begin{array}{ll}63 & 23.37\end{array}$ & 15322.37 & 629 & 982113.81 & -0.61 & -21.62 & -22.02 \\
\hline M495 & 634.27 & 1534.21 & 2183 & 981990.62 & 45.63 & -24.39 & -25.73 \\
\hline M496 & 7.53 & 15259.83 & 1908 & 982011.31 & 36.52 & -23.86 & -25.01 \\
\hline M497 & 8.63 & 15251.73 & 2024 & 982005.87 & 40.69 & -24.52 & -25.76 \\
\hline M498 & 6324.35 & $153 \quad 13.17$ & 655 & 982109.19 & -3.95 & -25.81 & -26.23 \\
\hline M499 & 6324.74 & 15311.64 & 662 & 982107.50 & -5.47 & -27.55 & -27.97 \\
\hline$M 500$ & 6324.55 & $153 \quad 10.05$ & 671 & 982104.62 & -7.21 & -29.61 & -30.04 \\
\hline$M 501$ & 6324.51 & $\begin{array}{ll}153 & 7.97\end{array}$ & 678 & 982104.69 & -6.48 & -29.11 & -29.55 \\
\hline M502 & $\begin{array}{ll}63 & 23.88\end{array}$ & $153 \quad 6.87$ & 696 & 982103.62 & -5.15 & -28.39 & -28.84 \\
\hline M503 & 6323.06 & $153 \quad 6.17$ & 709 & 982098.62 & -7.87 & -31.55 & -32.00 \\
\hline M504 & $\begin{array}{ll}63 & 22.47\end{array}$ & $153 \quad 4.73$ & 725 & 982097.50 & -6.76 & -30.98 & -31.45 \\
\hline M506 & $62 \quad 42.31$ & $153 \quad 7.79$ & 2629 & 981918.31 & 42.20 & -42.38 & -43.99 \\
\hline M507 & 6244.56 & $153 \quad 2.31$ & 2650 & 981917.12 & 40.18 & -44.15 & -45.76 \\
\hline
\end{tabular}


Table 1 (con.)

\begin{tabular}{|c|c|c|c|c|c|c|c|}
\hline 100 & Lat1tude & Long1tude & $\begin{array}{l}\text { Elev }_{\dot{\alpha}} \\
(f t)^{2}\end{array}$ & Observed & FAA & $C B A(2.62)$ & $\operatorname{CBA}(2.67$ \\
\hline M508 & 6246.71 & 15254.70 & 1955 & 981967.31 & 22.45 & -41.89 & -43.12 \\
\hline M509 & 6249.54 & 15255.97 & 1741 & 981980.62 & 12.10 & -45.78 & -46.88 \\
\hline$M 510$ & $62 \quad 52.69$ & $152 \quad 56.78$ & 1540 & 982000.12 & 8.81 & -42.45 & -43.43 \\
\hline M511 & $62 \quad 55.28$ & 15259.66 & 1366 & 982017.37 & 6.56 & -39.03 & -39.90 \\
\hline MS12 & 6257.04 & $153 \quad 3.46$ & 1227 & 982032.87 & 6.87 & -33.67 & $-34,45$ \\
\hline M513 & 6258.70 & $153 \quad 9.77$ & 1115 & 982042.81 & 4.14 & -33.08 & -33.79 \\
\hline M5 14 & $63 \quad 1.34$ & 15252.34 & 1764 & 982012.37 & 31.66 & -26.56 & -27.67 \\
\hline M515 & 3.28 & 15249.15 & 1906 & 982007.31 & 37.41 & -24.51 & -25.70 \\
\hline M516 & $63 \quad 5.98$ & 15232.21 & 1891 & 982012.81 & 38.29 & -23.45 & -24.62 \\
\hline M517 & 6256.23 & 15232.17 & 2470 & 981949.19 & 41.04 & -40.25 & -41.80 \\
\hline M5 18 & 6255.31 & $152 \quad 23.69$ & 2465 & 981941.62 & 34.07 & -47.58 & -49.14 \\
\hline M519 & 1.48 & $152 \quad 33.97$ & 1915 & 982001.31 & 34.46 & -29.25 & -30.47 \\
\hline M520 & 6311.50 & 15321.35 & 1120 & 982070.62 & 16.77 & -19.13 & -19.81 \\
\hline M521 & $63 \quad 15.41$ & 15313.27 & 1414 & 982059.69 & 28.80 & -16.34 & -17.20 \\
\hline M522 & $63 \quad 15,46$ & 15332.60 & 600 & 982107,81 & -0.57 & -20.62 & -20.16 \\
\hline M523 & $63 \quad 19.82$ & $.153 \quad 21.56$ & 630 & 982109.81 & -0.17 & -21.22 & -21.62 \\
\hline M524 & 6327.90 & $153 \quad 9.92$ & 1450 & 982058.62 & 15.92 & -30.02 & -30.90 \\
\hline M525 & 6330.41 & 9.10 & 2478 & 981987.12 & 38.16 & -36.00 & -37.42 \\
\hline M526 & $63 \quad 32.47$ & 7.01 & 2526 & 981981.87 & 34.96 & -40.13 & -41.56 \\
\hline M527 & $63 \quad 33.99$ & 5.33 & 2883 & 981964.13 & 48.89 & -32.96 & -34.52 \\
\hline M528 & $63 \quad 37.87$ & 4.55 & 1853 & 982049.37 & 32.64 & -26.41 & -27.54 \\
\hline M529 & $6339.7 \mathrm{l}$ & 2.59 & 1587 & 982067.81 & 23.78 & -27.79 & -28.77 \\
\hline M530 & 6342.22 & 0.62 & 1924 & 982049.87 & 34.53 & -26.62 & -27.79 \\
\hline M531 & 6330.27 & 15251.66 & 808 & 982092.19 & -13.68 & -40.67 & -41.18 \\
\hline M532 & 6323.99 & 15234.17 & 1042 & 982089.63 & 13,34 & -21.47 & -22.13 \\
\hline M533 & $63 \quad 12.53$ & 15244.45 & 1229 & 982059.81 & 15.05 & -25.98 & -26.76 \\
\hline M534 & $63 \quad 14.70$ & 15255.50 & 917 & 982080.81 & 3.95 & -26.65 & -27.23 \\
\hline M535 & 6343.04 & 15336.02 & 2271 & 982039.81 & 56.08 & -14.69 & -16.04 \\
\hline$M 536$ & 6343.03 & 15345.85 & 2907 & 981996.12 & 72.32 & -10.41 & -11.99 \\
\hline M537 & $63 \quad 38.36$ & 15352.91 & 2685 & 982005.69 & 66.58 & -9.91 & -11.37 \\
\hline M538 & 6335.95 & $153 \quad 57.37$ & 2496 & 982020.81 & 66.75 & -11.66 & -13.16 \\
\hline M539 & 6326.66 & 1547.87 & 2670 & 981993.50 & 67.15 & -12.89 & -14.42 \\
\hline M540 & $63 \quad 32.53$ & 3.85 & 2510 & 982014.81 & 66.31 & -8.96 & -10.40 \\
\hline M541 & 6320.03 & $154 \quad 26.17$ & 2265 & 982013.87 & 57.47 & -11.83 & -13.15 \\
\hline$M 542$ & $63 \quad 16.42$ & 15433.08 & 2767 & 981968.81 & 63.98 & -11.22 & -12.66 \\
\hline M543 & $63 \quad 12.76$ & 15441.18 & 1932 & 982019.69 & 40.77 & -13.81 & -14.85 \\
\hline MS44 & 639.31 & 15451.79 & 1481 & 982051.62 & 34.39 & -10.38 & -11.23 \\
\hline M545 & $63 \quad 3.80$ & 7.03 & 1856 & 982017.50 & 42.33 & -10.16 & -11.16 \\
\hline M546 & $\begin{array}{ll}63 & 0.87\end{array}$ & 15327.15 & 817 & 982071.87 & 2.61 & -24.37 & -24.88 \\
\hline M547 & 4.07 & 15338.11 & 648 & 982094.12 & 5.02 & -16.63 & -17.04 \\
\hline M548 & 0.50 & $153 \quad 46.71$ & 601 & 982087.12 & -2.05 & -22.14 & -22.52 \\
\hline M549 & $62 \quad 27.84$ & $153 \quad 44.35$ & 3216 & 981866.31 & 63.24 & -35.81 & -37.70 \\
\hline M550 & $62 \quad 25.77$ & $153 \quad 53.26$ & 4702 & 981769.87 & 109.27 & -25.07 & -27.64 \\
\hline M551 & $62 \quad 23.07$ & 15354.65 & 4010 & 981821.50 & 99.14 & -26.00 & -28.39 \\
\hline M552 & 6224.92 & 15359.63 & 4657 & 981773.19 & 109.36 & -21.63 & -24.13 \\
\hline M553 & $\begin{array}{ll}62 & 20.87\end{array}$ & $154 \quad 11.24$ & 2348 & 981927.00 & 51.06 & -19.13 & -20.47 \\
\hline M554 & $62 \quad 23.03$ & $154 \quad 13.00$ & 2047 & 981949.50 & 42.55 & -23.11 & -24.36 \\
\hline
\end{tabular}


Table 1 (con.)

\begin{tabular}{|c|c|c|c|c|c|c|c|}
\hline Station & Latitude & Longltude & $\begin{array}{l}\text { Elev } \\
(E t)\end{array}$ & Observed & FAA & $\operatorname{CBA}(2.62)$ & $\mathrm{CBA}(2.67)$ \\
\hline M555 & 6226.07 & 15414.15 & 1662 & 981962.31 & 15.31 & -39.18 & -40.22 \\
\hline M556 & 6231.83 & 15357.21 & 1333 & 982007.12 & 22.05 & -22.17 & -23.02 \\
\hline M557 & $62 \quad 33.46$ & $154 \quad 2.22$ & 1126 & 982025.62 & 19.11 & -18.36 & -19.08 \\
\hline M558 & $62 \quad 35.67$ & $154 \quad 5.60$ & 978 & 982041.87 & 18.68 & -13.92 & -14.54 \\
\hline M559 & $62 \quad 37.87$ & 1546.98 & 877 & 982050.38 & 15,04 & -14.21 & -14.76 \\
\hline M560 & 6240.19 & 7.61 & 773 & 982053.19 & 5.16 & -20.65 & $-2 L .14$ \\
\hline M561 & $62 \quad 42.18$ & $154 \quad 20.07$ & 686 & 982051.31 & -7.42 & -30.33 & -30.77 \\
\hline$M 562$ & 6243.68 & 15413.01 & 614 & 982054.37 & -12.89 & -33.40 & -33.79 \\
\hline M563 & $62 \quad 44.81$ & $154 \quad 16.73$ & 555 & 982066.87 & -7.36 & -25.90 & -26.26 \\
\hline$M 564$ & 6246.38 & 15420.11 & 500 & 982081.81 & 0.49 & -16.21 & -16.53 \\
\hline$M 565$ & $62 \quad 41.25$ & $153 \quad 33.83$ & 1613 & 981994.87 & 24.55 & -27.89 & -28.89 \\
\hline M566 & 6244.29 & 15342.01 & 979 & 982051.37 & 17.74 & -14.94 & -15.57 \\
\hline M567 & 6248.63 & 15344.22 & 818 & 982072.81 & 18.61 & -8.68 & -9.20 \\
\hline M568 & $62 \quad 51.33$ & 15347.14 & 671 & 982081.31 & 9.97 & -12.45 & -12.88 \\
\hline M569 & 6255.16 & 15348.57 & 620 & 982082,81 & 1.95 & -18.77 & -19.16 \\
\hline M570 & 6258.57 & 15352.70 & 566 & 982087.12 & -2.97 & -21.89 & -22.25 \\
\hline M57i & 6259.10 & 1542.99 & 507 & 982096.19 & -0.11 & -17.05 & -17.37 \\
\hline$M 572$ & 6257.48 & 15422.70 & 442 & 982096.87 & -3.51 & -18.28 & -18.56 \\
\hline$M 573$ & 6249.56 & 154.24 .16 & 473 & 982092.37 & $4.6 \mathrm{I}$ & $-11,19$ & -11.49 \\
\hline
\end{tabular}

